\title{
High Magnetic Field Behaviour of the Triangular Lattice Antiferromagnet, $\mathrm{CuFeO}_{2}$
}

\author{
O. A. Petrenko ${ }^{1,2}$, G. Balakrishnan², M. R. Lees², D. McK Paul ${ }^{2}$ and A. Hoser ${ }^{3}$ \\ ${ }^{1}$ ISIS Facility, Rutherford Appleton Laboratory, Chilton, Didcot, OX11 0QX, UK \\ ${ }^{2}$ University of Warwick, Department of Physics, Coventry, CV4 7AL, UK \\ ${ }^{3}$ Hahn-Meitner-Institut, Glienicker Straße 100, 14109 Berlin, Germany
}

\begin{abstract}
The high magnetic field behaviour of the triangular lattice antiferromagnet $\mathrm{CuFeO}_{2}$ is studied using single crystal neutron diffraction measurements in a field of up to $14.5 \mathrm{~T}$ and also by magnetisation measurements in a field of up to $12 \mathrm{~T}$. At low temperature, two well-defined first order magnetic phase transitions are found in this range of applied magnetic field $(H \| c)$ : at $H_{c 1}=7.6(3) / 7.1(3) \mathrm{T}$ and $H_{c 2}=13.2(1) / 12.7(1) \mathrm{T}$ when ramping the field up/down. In a field above $H_{c 2}$ the magnetic Bragg peaks show unusual history dependence. In zero field $T_{N 1}=14.2(1) \mathrm{K}$ separates a high temperature paramagnetic and an intermediate incommensurate structure, while $T_{N 2}=11.1(3) \mathrm{K}$ divides an incommensurate phase from the low-temperature 4-sublattice ground state. The ordering temperature $T_{N 1}$ is found to be almost field independent, while $T_{N 2}$ decreases noticeably in applied field. The magnetic phase diagram is discussed in terms of the interactions between an applied magnetic field and the highly frustrated magnetic structure of $\mathrm{CuFeO}_{2}$.
\end{abstract}

PACS numbers: 75.25.+z, 75.30.Kz, 75.50.Ee, 75.60.Ej

\section{INTRODUCTION}

Compounds of the $\mathrm{ABO}_{2}$-type with the delafossite structure provide good examples of antiferromagnets on a triangular lattice, presenting the opportunity to study the influence of geometrical frustration on these magnetic systems 1 . Unlike the well-studied quasi one-dimensional $\mathrm{ABX}_{3}$-type hexagonal antiferromagnets (where $\mathrm{A}$ is an alkali metal, $\mathrm{B}$ is a bivalent metal of the $3 d$ group and $\mathrm{X}$ is a halogen)2 2 , the $\mathrm{ABO}_{2}$ materials are mostly quasi two-dimensional and are highly frustrated between neighbouring triangular layers as well as within a layer. The study of the magnetic properties of $\mathrm{ABO}_{2}$ compounds has been somewhat hampered by the absence of good quality single crystals. However, recent progress in crystal growth for one of the members of the delafossite-family, $\mathrm{CuFeO}_{2} \mathrm{3}$, has revived the interest in this compound and generated a "second wave" of publications dealing with its magnetic properties 1 .

Despite recent efforts, the situation regarding the magnetic properties of $\mathrm{CuFeO}_{2}$ is far from clear. An earlier powder neutron diffraction study 8 claimed to observe two successive magnetic transitions at $T_{N 1}=16 \mathrm{~K}$ and $T_{N 2}=10 \mathrm{~K}$ to a collinear spin structure with a monoclinic unit cell $(\sqrt{7} a \times \sqrt{7} a \times 2 c$, five spins in a layer $)$ and an orthorhombic unit cell $(\sqrt{3} a \times 2 a \times 2 c$, four spins in a layer) respectivelye. All the spins were found to be parallel to the $c$-axis and an Ising nature for the exchange interactions has been suggested, although that would be unusual for the ${ }^{6} S$ state of $\mathrm{Fe}^{3+}$ ions. Further neutron powder diffraction measurements as well as the single crystal susceptibility and Mössbauer effect studies 10 also showed two successive phase transitions, however it was suggested that the intermediate temperature (IT) phase is a partially disordered phase, where $1 / 5$ of the magnetic moments remain paramagnetic. The presence of disordered spins in the IT phase agrees with the heat capacity data11, but contradicts the simple mean-field theory for the Ising antiferromagnet on a triangular lattice which suggests a partially disordered phase, where one third of the spins are paramagnetic, rather than one fifth.

The most interesting effects have been found when $\mathrm{CuFeO}_{2}$ is placed in a magnetic field. As many as five spin-flop like magnetisation anomalies have been observed for a field applied along the $c$-axis (at 8, 13, 22, 26, 42 and $70 \mathrm{~T}$ ) ${ }^{12}$, while for $H \perp c$, a phase transition has been found at $24 \mathrm{~T}$. Monte Carlo simulation results have suggested that in order for a collinear structure to be stable in zero field 10 and also undergo several phase transitions in applied field, the second neighbour exchange, $J_{2}$, as well as the third neighbour exchange, $J_{3}$, have to be unusually strong in comparison with the main nearest-neighbour exchange interaction $J_{1}: J_{2} / J_{1}=0.5$ and $J_{3} / J_{1}=0.75$. Although a theoretical model with such parameters produces a somewhat similar magnetisation curve, comparable values of the exchange interactions $J_{1}$ and $J_{3}$ for magnetic moments separated by twice the distance seems to be suspicious. In fact, weak interplane interactions which have been neglected in this study might play an important role in the formation of the long range magnetic order.

The exact magnetic structure of the IT phase is still a subject of discussion. Submillimeter wave ESR measurementst have discovered an easy-plane antiferromagnetic resonance mode which could not be explained in the partially disordered model suggested by previous powder neutron diffraction experiments. Becently the first single crystal neutron diffraction study has shown that between $T_{N 1}$ and $T_{N 2}$ a quasi-long range amplitudemodulated magnetic structure exists. This structure has an incommensurate, temperature dependent, propagation vector $(q q 0)$ with $q$ ranging from 0.19 to 0.22 .

The crystal structure of $\mathrm{CuFeO}_{2}$ belongs to the space group $R \overline{3} m$ with $a=3.03 \AA$ and $c=17.09 \AA$ in the hexagonal description. The structure consists of hexagonal lay- 
ers of $\mathrm{Cu}, \mathrm{O}$ and $\mathrm{Fe}$ with a stacking sequence of $\mathrm{O}^{2-}$ $\mathrm{Fe}^{3+}-\mathrm{O}^{2-}-\mathrm{Cu}^{+}-\mathrm{O}^{2-}-\mathrm{Fe}^{3+}-\mathrm{O}^{2-}$ along the $c$ axis, where the triangular lattice of magnetic $\mathrm{Fe}^{3+}$ ions are separated by nonmagnetic ionic layers of $\mathrm{Cu}^{+}$and $\mathrm{O}^{2-}$, see Fig. 11. Therefore, a quasi two-dimensional character for the magnetic exchange interactions is the most likely situation, however no actual measurements of the exchange parameters have been reported so far and the extent of the low-dimensionality of the system remains unknown.

In this paper we report on single crystal heat capacity, magnetisation and neutron diffraction measurements. Our main experimental efforts were focused on the properties of the low-temperature (LT) collinear phase in a magnetic field applied either parallel or perpendicular to the caxis. We find that contrary to previous suggestions 612 a field-induced phase transition from a collinear 4-sublattice state to a 5-sublattice state occurs only when an applied magnetic field exceeds the value of the second critical field, $H_{c 2} \approx 13.2 \mathrm{~T}$, rather than the first one, $H_{c 1} \approx 7.6 \mathrm{~T}$. The exact nature of the phase transition at $H_{c 2}$, however, remains unclear.

\section{EXPERIMENTAL DETAILS}

Polycrystalline $\mathrm{CuFeO}_{2}$ was synthesised starting from stoichiometric quantities of the oxides $\mathrm{CuO}$ and $\mathrm{Fe}_{2} \mathrm{O}_{3}$. A mixture of the oxides was first reacted at $800^{\circ} \mathrm{C}$ for 15 hours in air followed by 24 hours in flowing nitrogen gas with intermediate grindings. Rods of diameter 8-10 mm and length $8-10 \mathrm{~cm}$ were then isostatically pressed from the reacted powder, and sintered at $950^{\circ} \mathrm{C}$ in flowing nitrogen gas. Single crystals of $\mathrm{CuFeO}_{2}$ were grown by the floating zone method using a four mirror image furnace (model CSI FZ-T-10000-H-IV-VPS). The rods were rotated at $30 \mathrm{rpm}$ and the growth was carried out at $1 \mathrm{~mm} / \mathrm{h}$, in 1 atmosphere of argon gas with a flow of $0.3 \mathrm{l} / \mathrm{min}$. The crystals produced had facets and the growth axis was found to be inclined to the $c$-axis at approximately 10 degrees.

The specific heat measurements in zero field were made in the temperature range 2 to $20 \mathrm{~K}$ using a standard heat pulse-relaxation method and from 20 to $305 \mathrm{~K}$ using an adiabatic technique. The data were collected while warming the sample.

The magnetisation data, $M$ vs $H$ and $M$ vs $T$, were collected using an Oxford Instruments vibrating sample magnetometer in an applied field of up to $12 \mathrm{~T}$. For both the specific heat and magnetisation measurements we have used small ( 5 to $40 \mathrm{mg}$ ) plate-like samples that were cut from the crystal used for neutron scattering experiments. The absolute accuracy of the magnetisation measurements was better than $1 \%$. The crystals were aligned with an accuracy of $1-2^{\circ}$.

The neutron scattering measurements were carried out using the E1 triple-axis spectrometer at the Berlin Neu- tron Scattering Center, HMI, Germany. The spectrometer was used in the double-axis mode, with a pyrolytic graphite (002) monochromator providing a neutron wavelength of $2.42 \AA$. A pyrolytic graphite filter was installed in the incident beam to remove higher order contamination.

The single crystal sample of almost cylindrical shape (13.5 $\mathrm{mm}$ in length and $6.5 \mathrm{~mm}$ in diameter) was aligned in a vertical field cryomagnet VM-1 in two different orientations. In the first case the horizontal scattering plane contained the $(h k 0)$ reflections and in the second case the $(h h l)$ reflections. The horizontal collimation was 40'-80'40 ', typical counting times were 1 to 5 seconds at each point.

\section{EXPERIMENTAL RESULTS}

\section{A. Low temperature specific heat data}

The low-temperature specific heat measurements of the $\mathrm{CuFeO}_{2}$ single crystal (see Fig. 2) show a remarkable difference between the two magnetic phase transitions. The first transition into the IT phase is marked by a broad peak in the specific heat at a temperature of around $14 \mathrm{~K}$. The second transition into the LT phase is accompanied by a much sharper, more intense peak at $T \approx 11 \mathrm{~K}$.

Previous thermal analysis measurements 11 claimed that the magnetic entropy associated with the anomaly in the heat capacity around $T_{N 2}$ is attributable to the disordering of one-fifth of the $\mathrm{Fe}^{3+}$ spins. However, this conclusion seems to be based on an unreliable estimate of the heat capacity baseline (see Fig. 3 in Ref.11). The data presented here suggests that nearly one third of the $\mathrm{Fe}^{3+}$ spins are disordered by the time the temperature is increased to $T_{N 2}$. This observation is in good agreement with the value expected from the simple mean-field theory for an Ising antiferromagnet on a triangular lattice.

Our high-temperature specific heat data indicate that $\mathrm{CuFeO}_{2}$ has a relatively high Debye temperature, $\theta_{D} \approx$ $600 \mathrm{~K}$. Therefore, the phonon contribution to the specific heat at low temperatures is negligibly small compared to the magnetic contribution. The presence of short range magnetic correlations dominates the heat capacity at all temperatures up to $40 \mathrm{~K}$, well above the magnetic ordering temperature. In fact only about three quarters of the magnetic entropy is recovered at $T<T_{N 1}$, as shown on the inset to Fig. 2, while the remaining quarter of the entropy is spread over $T>T_{N 1}$. The lattice contribution to the entropy, which does not exceed $2 \%$ of the total entropy at all temperatures shown on Fig. 2, has been neglected in these calculations. 


\section{B. Magnetisation data}

The first order nature of the lower temperature phase transition is clear from the temperature dependence of the magnetisation data. $M_{H \| c}$ exhibits a dramatic jump at $T_{N 2}$, while a small kink at $T_{N 1}$ became obvious only after differentiation of the data (see Fig. 3).

An interesting feature was noticed when the sample was warmed and then cooled in an applied magnetic field. If the field is below a spin-flop phase transition at $H_{c 1}$, then the hysteresis in the magnetisation is evident only around $T_{N 2}$, while in a field above $H_{c 1}$ the magnetisation curves $M(T)$ for increasing and decreasing temperature are significantly different for all temperatures below $T_{N 2}$. These results are consistent with the neutron diffraction data (see below).

In a low applied field $\mathrm{CuFeO}_{2}$ is magnetically highly anisotropic. This can be seen from the $M$ vs $H$ measurements for $H \perp c$ and $H \| c$ (Fig. đ). Note that the experimentally observed ratio of the susceptibilities parallel and perpendicular to c, $\chi_{\|} / \chi_{\perp} \approx 0.12$ is inconsistent with the $120^{\circ}$ triangular magnetic structure, where the value of 0.5 is expected at zero temperature. The magnetic anisotropy decreases abruptly in a field above the spin-flop phase transition at $H_{c 1}=7.6(3) / 7.1(3) \mathrm{T}$, however it does not disappear completely as expected for a simple three-dimensional collinear antiferromagnet. Even at $H=10 \mathrm{~T} M_{H \| c}$ is still only $73 \%$ of $M_{H \perp c}$. It would be very interesting to see whether $M_{H \perp c}$ and $M_{H \| c}$ do become equal in some higher field (above the second and possibly third transition fields, $H_{c 2}$ and $H_{c 3}$ ) or if they remain different, as has been found in the easyplane $\mathrm{ABX}_{3}$-type antiferromagnets 13 .

\section{Neutron diffraction data}

\section{1. (hhl) scattering plane, $H \perp c$.}

In zero magnetic field the LT magnetic structure is characterised by the appearance of magnetic Bragg reflections at the positions $\left(\frac{2 n+1}{4} \frac{2 n+1}{4} \frac{6 m+3}{2}\right)$, where $n$ and $m$ are integers, in accordance with observations by Mitsuda et al.J. The application of a magnetic field up to 14.5 $\mathrm{T}$ along a direction perpendicular to the scattering plane does not change visibly the position, intensity or width of these peaks - the 4-sublattice magnetic structure below $T_{N 2}$ remains unchanged by a field applied perpendicular to the $c$-axis.

At an intermediate temperature, $T_{N 2}<T<T_{N 1}$, the 4-sublattice magnetic structure is replaced by an incommensurate phase, in which the magnetic Bragg reflections occur at positions $\left(q q \frac{6 m+3}{2}\right)$, where $m$ is an integer and the value of $q$ is temperature dependent and ranges from 0.19 to 0.225 . In the vicinity of $T_{N 2}$ and $T_{N 1}$ the width of the magnetic peaks is not resolution limited, which suggests the absence of true long-range magnetic ordering. However at all temperatures away from the phase boundaries, that is at $11 \mathrm{~K}<T<13 \mathrm{~K}$, the width of the magnetic peaks is resolution limited. It is worth noting, that at $T=10.5 \mathrm{~K}$, both commensurate and incommensurate peaks are present in the diffraction pattern. These results are in general agreement with the previous findings of Mitsuda et al.J.

As with the LT phase, the application of a magnetic field $H \perp c$ does not affect the characteristics of the IT phase. It only marginally decreases the temperature of the incommensurate to commensurate phase transition, $T_{N 2}$, lowering it to $10 \mathrm{~K}$ in $14.5 \mathrm{~T}$.

\section{2. (hk0) scattering plane, $H \| c$.}

In zero magnetic field no purely magnetic reflections have been found in the $(h k 0)$ scattering plane. On the application of a magnetic field above $13 \mathrm{~T}$ along the $c$ axis at low temperature, scans in the $(h k 0)$ plane of the reciprocal lattice revealed new magnetic Bragg peaks at position $\left(\frac{n}{5} \frac{m}{5} 0\right)$, where $n$ and $m$ are nonzero integers and $n+m$ is even. This observation suggests that the magnetic field induces a transition from a purely antiferromagnetic ordering in zero field to an ordering with a ferromagnetic component along the $c$-axis.

The transition to the high-field phase is accompanied by a significant hysteresis, of about $0.5 \mathrm{~T}$. The transition field and also the hysteresis width gradually decrease as the temperature increases, though even at $T=8.1 \mathrm{~K}$, a temperature relatively close to the phase transition point $T_{N 2}$, the hysteresis amounts to only $0.2 \mathrm{~T}$. We have found no indication of any short range order within the high field induced magnetic phase - the widths of the new magnetic peaks are resolution limited. The field dependence of the integrated intensity of one of the peaks, $\left(\frac{1}{5} \frac{1}{5} 0\right)$, is shown on Fig. 5. The rest of the $\left(\frac{n}{5} \frac{n}{5} 0\right)$-type peaks behave similarly.

The temperature dependence of the intensity, width and positions of the $\left(\frac{n}{5} \frac{n}{5} 0\right)$ peaks show remarkable variations in behaviour for different values of $n$. Fig. 6] shows the temperature dependence of the integrated intensity and also of the position in the reciprocal lattice, $n$, for the two such peaks, with $n \approx 1$ and $n \approx 2$. Initially the sample was cooled down to $2 \mathrm{~K}$ in a zero field, and then the magnetic field was increased to $14.5 \mathrm{~T}$. Warming the sample above $T_{N 2}$ in the magnetic field results in a shift of the peak positions from $\left(\frac{1}{5} \frac{1}{5} 0\right)$ to $(0.225,0.225,0)$ and from $\left(\frac{2}{5} \frac{2}{5} 0\right)$ to $(0.385,0.385,0)$. The higher temperature peaks are still located along the $(h h 0)$ direction. When the sample was cooled back down to $2 \mathrm{~K}$ in an applied magnetic field, the first peak with $n \approx 1$ and originally in the $\left(\frac{1}{5} \frac{1}{5} 0\right)$ position has only regained approximately half its full integrated intensity and has remained in the incommensurate position $(0.203(1), 0.203(1), 0)$. For the second reflection with $n \approx 2$ the loss of integrated in- 
tensity was significantly smaller, while the change of its position from commensurate $\left(\frac{2}{5} \frac{2}{5} 0\right)$ to incommensurate $(0.396(1), 0.396(1), 0)$ was still evident (see Fig. 6). Other peaks of $\left(\frac{n}{5} \frac{m}{5} 0\right)$-type demonstrate similar history dependence, e.g. as a result of thermal cycling in a field, the $\left(\frac{3}{5} \frac{3}{5} 0\right)$ peak shifts its position to $(0.604,0.604,0)$ and the $\left(\frac{4}{5} \frac{4}{5} 0\right)$ peak to $(0.795,0.795,0)$.

At temperatures below the magnetic ordering an unusual behaviour of the (110) peak has been observed. In zero magnetic field the intensity of this peak increases in the IT phase and then remains almost constant in the LT phase. When the crystal was aligned with the $c$-axis in the horizontal plane the overall gain in intensity amounted to nearly $10 \%$ of the nuclear intensity at $T>T_{N 1}$. A much more pronounced gain in intensity of the same peak has been seen when the crystal was aligned with the $c$-axis vertical (see Fig. 7). In this case the integrated intensity at $T=2 \mathrm{~K}$ is more than doubled compared to the intensity at $T>T_{N 1}$. An abrupt change in slope in the intensity versus temperature curve is clearly visible at $T=T_{N 2}$. In an applied magnetic field this kink gradually disappears, so that at $H=14.5 \mathrm{~T}$ no anomaly associated with $T_{N 2}$ is visible.

Mitsuda et al. have also detected a significant increase of the (110) peak intensity and have attributed this to a dramatic variation of the crystal mosaic, based on the fact that (110) is not present in the neutron powder datal. We note that the observed change in width of the (110) peak is only $7 \%$ and that the other possible explanations of this effect could be either multiple scattering involving magnetic peaks or a truly magnetic nature of the (100) peak. The former suggestion is complicated by the fact that the strongest observed magnetic peak is only half the magnitude of the gain in intensity of the (110) peak. The latter suggestion has to be dismissed on the basis of a polarised neutron scattering experiment. The results of this experiment show that for the (110) reflection the ratio of the neutron scattering intensities with the spin flipper on and off remains practically unchanged when passing through the phase transition temperatures, $T_{N 1}$ and $T_{N 2}$. Therefore further investigations of the nature of the low-temperature behaviour of the (110) peak are required.

\section{DISCUSSION AND CONCLUSIONS}

We have noted in the Introduction that it is fairly unusual for the ${ }^{6} S$ state of $\mathrm{Fe}^{3+}$ ions to develop exchange interactions of the Ising-type, suggested by the previous investigations 9 . On the other hand, it is also hard to imagine how a magnetic system with more degrees of freedom (Heisenberg or XY-type) could demonstrate as many as six first order-type phase transition in an applied magnetic field. The most likely answer to this puzzle is the presence in $\mathrm{CuFeO}_{2}$ of sufficiently strong competition between the easy-axis anisotropy and the exchange inter- actions. Therefore it is essential to describe the magnetic phase diagram of $\mathrm{CuFeO}_{2}$ as accurately as possible and to compare it to similar systems in order to place $\mathrm{CuFeO}_{2}$ into the general picture of highly frustrated magnets.

An overall phase diagram of $\mathrm{CuFeO}_{2}$ for a magnetic field applied along the hexagonal axis is shown in Fig. 8. It contains a high temperature paramagnetic phase, an intermediate temperature incommensurate magnetic phase and also three different commensurate magnetic phases at low temperature. Contrary to previous results magnetic order in the IT phase at all temperatures except very close to the phase boundaries. At low temperature a magnetic field above $H_{c 2} \approx 13.2 \mathrm{~T}$ induces a magnetic state characterized by an ordering wavevector $\mathbf{q}=\left(\frac{1}{5} \frac{1}{5} 0\right)$.

The nature of the lower-field phase transition at $H_{c 2} \approx$ $7.6 \mathrm{~T}$ is less clear. In order to judge whether it involves Heisenberg degrees of freedom or corresponds to an Ising type rearrangement of the $\mathbf{q}=\left(\frac{1}{4} \frac{1}{4} \frac{3}{2}\right)$-structure, we would need to make a survey of all the magnetic peak intensities. To do this it would be necessary to measure scattering in the $(h h l)$ plane while the magnetic field is applied along the $c$-axis, which means that the field must be applied in the horizontal plane. No such facilities are currently available.

Surprisingly enough the magnetic phase diagram of $\mathrm{CuFeO}_{2}$ more closely resembles the $H-T$ phase diagrams of the rare-earth intermetallic compound $\$ 18$, rather than those of the $\mathrm{ABX}_{3}$-type stacked triangular Ising antiferromagnets19, such as $\mathrm{CsCoBr}_{3}$ and $\mathrm{CsCoCl}_{3}$. In particular, an incommensurate magnetic ordering occurring in rare-earth intermetallic compounds at $T_{N 1}$ exhibits at some lower temperature a transition to a simpler structure with a shorter period. This happens because in the case of axially anisotropic systems, an incommensurate or long-periodgommensurate modulated structure is unstable at $0 \mathrm{~K} 1 \mathrm{~S}$. At lower temperature an applied magnetic field lowers the free energy of the incommensurate phases more rapidly than that of the simple commensurate phases, therefore a reappearance of the incommensurability is often observed in these compounds in a magnetic field.

Splitting of $T_{N}$ is also a common feature for $\mathrm{ABX}_{3}$-type stacked triangular Heisenberg antiferromagnets with an easy-axis type of anisotropyl, however their behaviour in an applied magnetic field are profoundly different to that of $\mathrm{CuFeO}_{2}$. Their magnetisation curves demonstrate only one spin-flop transition at a critical field $H_{c}$ for a field applied along the hexagonal axis and the absence of any transitions for the field applied perpendicular to this axis. In addition, in $\mathrm{ABX}_{3}$-type systems the application of a magnetic field results in a decrease of the temperature difference between two phase transition, $T_{N 1}-T_{N 2}$, while for $\mathrm{CuFeO}_{2}$ the effect is the opposite - an applied magnetic field stabilises an intermediate incommensurate structure.

In high magnetic field both magnetisation and neutron 
diffraction measurements show an unusual history dependence. Cooling the sample in a high magnetic field results in a higher net magnetisation and also in the locking of the magnetic peaks in the incommensurate positions.

We should also mention the possibility of a high sensitivity of the magnetic properties of $\mathrm{CuFeO}_{2}$ to the presence of chemical impurities 20 and to the sample preparation technique. The results of Mitsuda et al. appear to be somewhat sample dependent. Our own magnetisation measurements have revealed a significant ferromagnetic component in the net magnetisation of those single crystals that have been obtained from our early attempts of crystal growth, before the growth conditions were optimised. The results of measurements of magnetisation, heat capacity and neutron diffraction on optimally prepared crystals, however, are self-consistent and also sample independent.

In conclusion, we have presented the results of neutron elastic scattering measurements, heat capacity and magnetisation studies on a single crystal of $\mathrm{CuFeO}_{2}$, a quasi two-dimensional antiferromagnet on a triangular lattice. These measurements have allowed a detailed study of the unusual low temperature magnetic properties of $\mathrm{CuFeO}_{2}$ in an applied magnetic field of up to $14.5 \mathrm{~T}$.

\section{ACKNOWLEDGEMENTS}

This work was supported by the Engineering and Physical Sciences Research Council. O. P. acknowledges financial support by the Hahn-Meitner-Institut, Berlin.

${ }^{1}$ The list of $\mathrm{ABO}_{2}$-type compounds for which the magnetic properties have been studied to different degrees, include $\mathrm{ACrO}_{2}(\mathrm{~A}=\mathrm{Li}, \mathrm{Na}, \mathrm{K}, \mathrm{Cu}, \mathrm{Ag}$ and $\mathrm{Pd}), \mathrm{NaTiO}_{2}, \mathrm{LiNiO}_{2}$, $\mathrm{PdCoO}_{2}, \mathrm{CuFeO}_{2}, \mathrm{AgNiO}_{2}, \mathrm{CuNdO}_{2}$ and $\mathrm{CuMnO}_{2}$.

${ }^{2}$ M. F. Collins and O. A. Petrenko, Can. J. Phys. 75, 605 (1997).

3 T.-R. Zhao, M. Hasegawa and H. Takei, J. Cryst. Growth 166, 408 (1996); 154, 322 (1995).

${ }^{4}$ T. Fukuda, H. Nojiri, M. Motokawa, T. Asano, M. Mekata and Y. Ajiro, Physica B 246-247, 569 (1998).

${ }^{5}$ V. R. Galakhov, A. I. Poteryaev, E. Z. Kurmaev, V. I. Anisimov, St. Bartkowski, M. Neumann, Z. W. Lu, B. M. Klein and T.-R. Zhao, Phys. Rev. B 56, 4584 (1997).

${ }^{6}$ S. Mitsuda, T. Uno, M. Mase, H. Nojiri, K. Takashi, M. Motokawa and M. Arai, J. Phys. Chem. Solids 60, 1249 (1999).

${ }^{7}$ S. Mitsuda, N. Kasahara, T. Uno and M. Mase, J. Phys. Soc. Japan 67, 4026 (1998).

${ }^{8}$ S. Mitsuda, H. Yoshizawa, N. Yaguchi and M. Mekata, J. Phys. Soc. Japan 60, 1885 (1991).

${ }^{9}$ Although reporting apparently the same data as in Ref 8 , the authors M. Mekata et al. quote a different value for the lower temperature phase transition, $T_{N 2}=11 \mathrm{~K}$, in J. Mag. and Mag. Mat. 104-107, 823 (1992).

${ }^{10}$ M. Mekata, N. Yaguchi, T. Takagi, T. Sugino, S. Mitsuda, H. Yoshizawa, N. Hosoito and T. Shinjo, J. Phys. Soc. Japan 62, 4474 (1993).

${ }^{11}$ K. Takeda, K. Miyake, M. Hitaka, T. Kawae, N. Yaguchi and M. Mekata, J. Phys. Soc. Japan 63, 2017 (1994).

${ }^{12}$ Y. Ajiro, T. Asano, T. Takagi, M. Mekata, H. Aruga-Katori and T. Goto-Physica B 201 71 (1994).

${ }^{13}$ In $\mathrm{CsMnBr}_{3} 14$ and $\mathrm{RbMnBr}_{3} 15$ an experimentally observed anisotropy between the magnetisation when the field is applied along and perpendicular to the-axis has begn attributed to the influence of quantum 16 or thermal17 fluctuations, enhanced by the low-dimensional nature of the system.

${ }^{14}$ I. A. Zaliznyak, Solid State Comm. 84, 573 (1992).

15 A. G. Abanov and O. A. Petrenko, Phys. Rev. B 50, 6271 (1994).

16 S. I. Abarzhi, A. N. Bazhan, L. A. Prozorova and I. A. Zaliznyak, J. Phys.: Condens. Matter 4, 3307 (1992).

${ }^{17}$ P. Santini, Z. Domanski, J. Dong and P. Erdos, Phys. Rev. B 54, 6327 (1996).

18 D. Gignoux and D. Schmitt, Phys. Rev. B 48, 12682 (1993).

19 B. D. Gaulin, in Magnetic Systems with Competing Interactions, edited by H. T. Diep (World Scientific, Singapore), 286 (1994).

${ }^{20}$ Y. Ajiro, K. Hanasaki, T. Asano, T. Takagi, M. Mekata, H. Aruga-Katori and T. Goto, J. Phys. Soc. Japan 64, 3643 (1995).

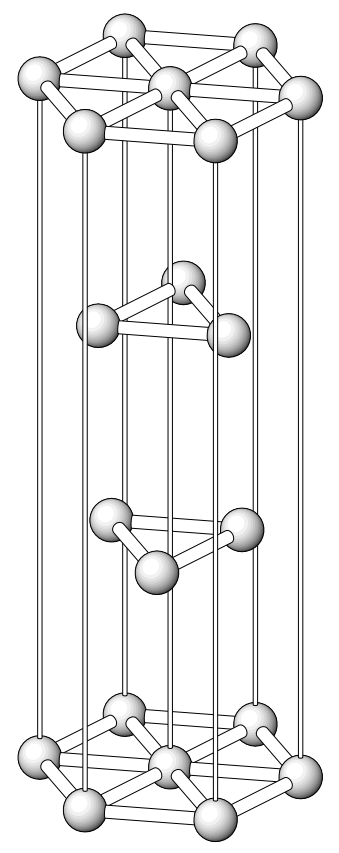


FIG. 1. Crystal structure of $\mathrm{CuFeO}_{2}$. Only $\mathrm{Fe}^{3+}$ magnetic ions are shown.

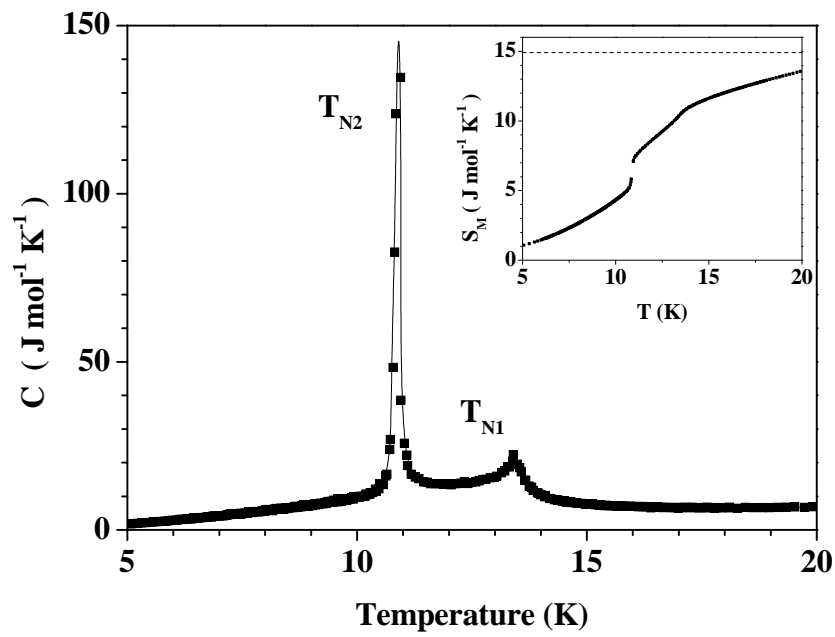

FIG. 2. Low-temperature heat capacity of $\mathrm{CuFeO}_{2}$ single crystal. Solid line is a guide for the eye. The inset shows the temperature dependence of the magnetic entropy, calculated as $S_{M}(T)=\int_{0}^{T} C / T d T$. The dashed line indicates the maximum magnetic entropy $R \ln (2 \mathrm{~S}+1)=14.90 \mathrm{~J} \mathrm{~mol}^{-1} \mathrm{~K}^{-1}$ for $\mathbf{S}=5 / 2$.

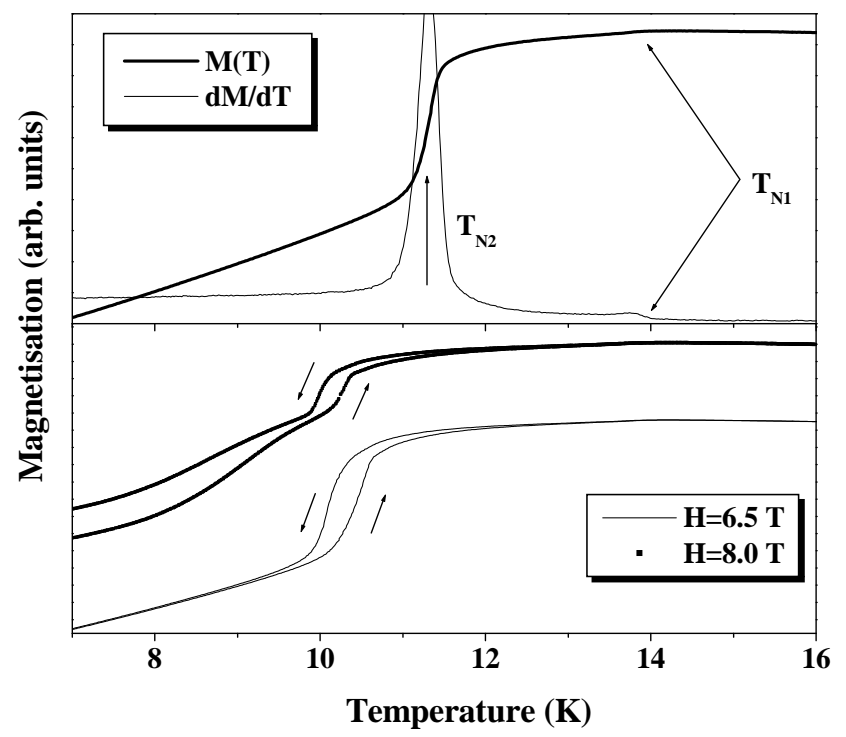

FIG. 3. Temperature dependence of the magnetisation $M_{H \|_{c}}$ (in arbitrary units) measured for a $\mathrm{CuFeO}_{2}$ single crystal at: $H=0.05 \mathrm{~T}$ while warming the sample (upper panel); $H=6.5 \mathrm{~T}$ and $H=8.0 \mathrm{~T}$ while warming and cooling the sample (lower panel).

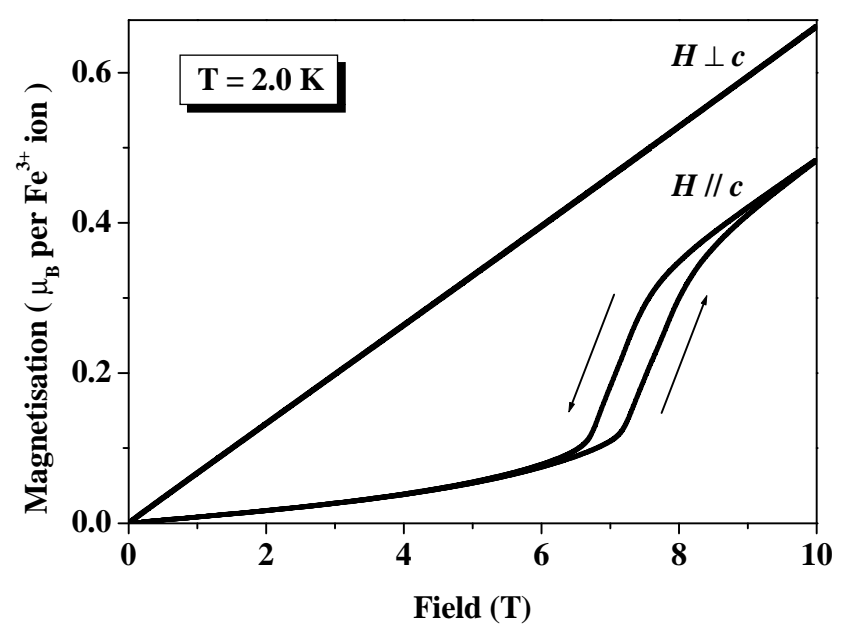

FIG. 4. Field dependence of the magnetisation at $T=2.0 \mathrm{~K}$ for a $\mathrm{CuFeO}_{2}$ single crystal.

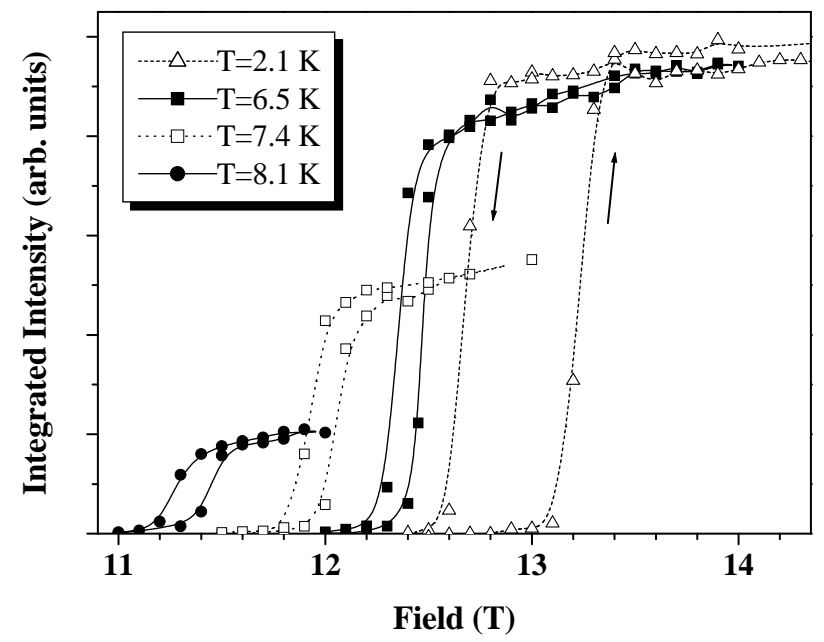

FIG. 5. Integrated intensity of the $\left(\frac{1}{5} \frac{1}{5} 0\right)$ peak of $\mathrm{CuFeO}_{2}$ in applied magnetic field, $H \| c$, at different temperatures.

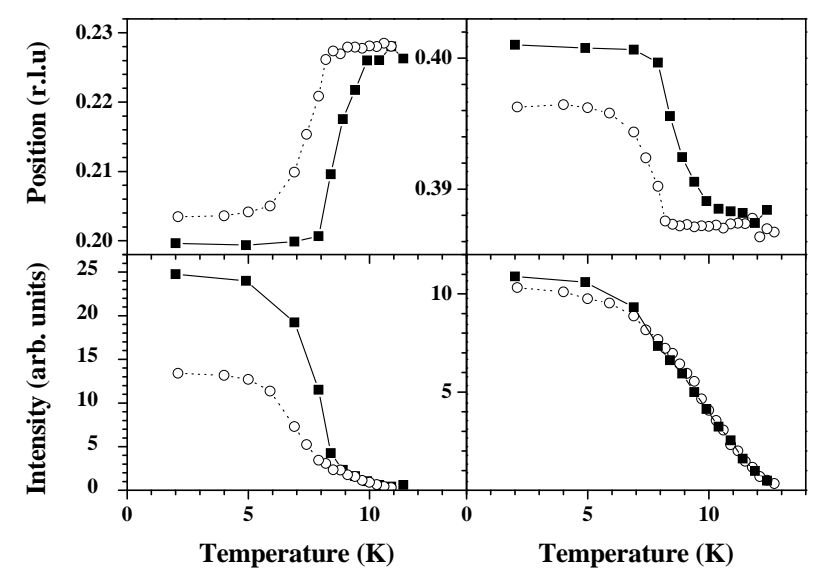


FIG. 6. Temperature dependence of the position, $n$, (upper panel) and the integrated intensity (lower panel) of two magnetic peaks of type $\left(\frac{n}{5} \frac{n}{5} 0\right)$ of $\mathrm{CuFeO}_{2}$ in an applied magnetic field of $14.5 \mathrm{~T}, H \| c$. Solid and open symbols correspond to warming and cooling of the sample respectively.

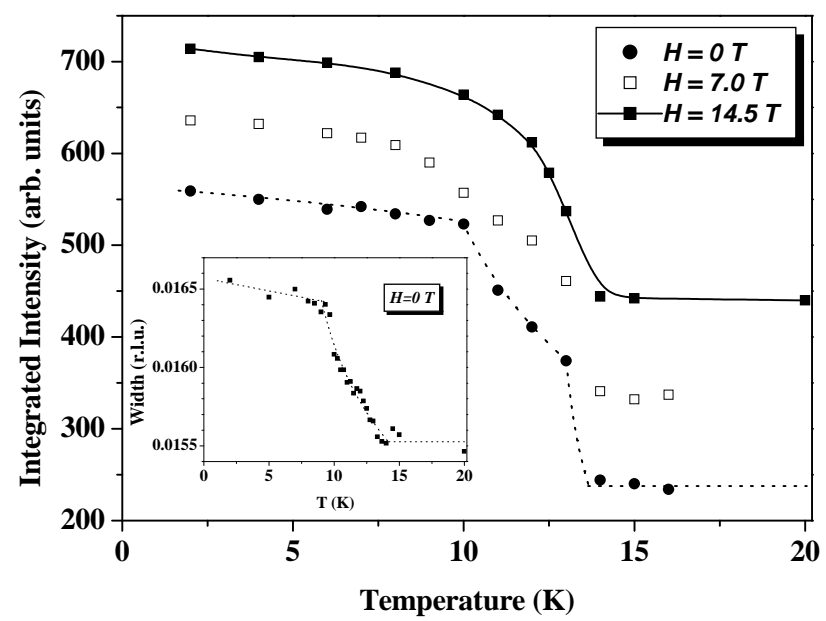

FIG. 7. Temperature dependence of the intensity of (110) peak in $\mathrm{CuFeO}_{2}$ in applied magnetic field, $H \| c$. For clarity, the curves for $H=7 \mathrm{~T}$ and $H=14.5 \mathrm{~T}$ have been offset by 100 and 200 respectively. The inset shows the temperature dependence of the linewidth of this peak in zero field.

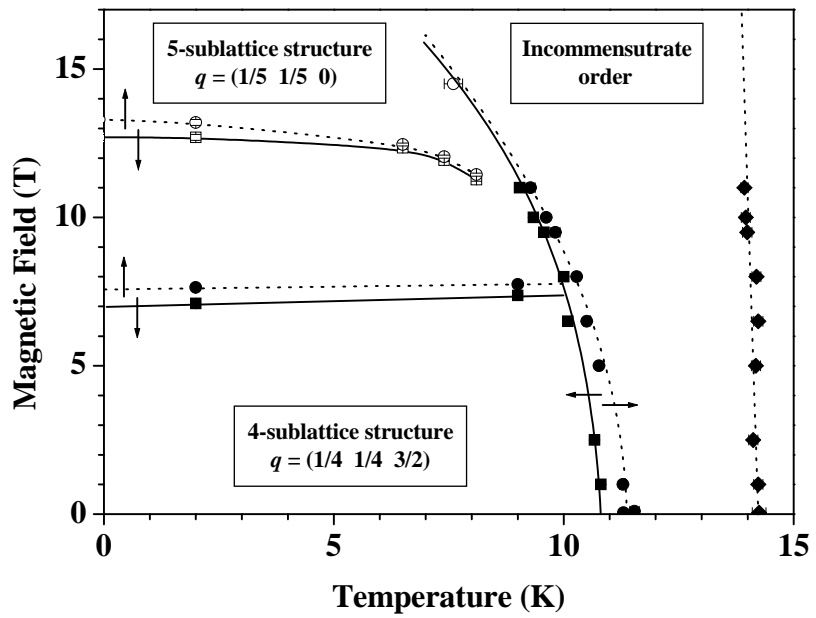

FIG. 8. Magnetic phase diagram of $\mathrm{CuFeO}_{2}, H \| c$, as obtained from magnetisation (solid symbols) and neutron diffraction (open symbols) data. Solid and dashed lines correspond to decreasing and increasing temperature or magnetic field respectively. Only the transition from paramagnetic to an incommensurate phase is of second order. The rest of the transitions are of first order with a well pronounced hysteresis. 